\title{
Nivel de pensamiento crítico de los profesionales de enfermería en la técnica de manejo de cateterismo vesical en los servicios de emergencia, hospitalización de medicina y cirugía del Hospital Vitarte
}

\author{
Critical thinking level of nurses in management technique catheterization in the emergency services, \\ hospitalization, medical and surgical Vitarte Hospital
}

Luz Yanet Barboza Núñez', Ruth Yenny Chura Muñuico²

\begin{abstract}
RESUMEN
Objetivo: Determinar el nivel de pensamiento crítico de los profesionales de enfermería en la técnica de manejo de cateterismo vesical en los servicios de emergencia, hospitalización de medicina y cirugía del Hospital Vitarte. Material y Métodos: El diseño de investigación fue cuantitativo, descriptivo, de corte transversal. La muestra estuvo conformada por 45 enfermeras (os) de los servicios de emergencia (25), hospitalización de medicina (12) y cirugía (8), del Hospital Vitarte. Se utilizó el instrumento, "Cuestionario de Pensamiento Crítico", que constó de 39 preguntas específicas, el mismo que fue validado por expertos. La prueba de confiabilidad fue de 0,803 según el Coeficiente alfa de Cronbach. Resultados: El estudio evidencia que del 100\% de la muestra, el $64,4 \%$ de los profesionales de enfermería, alcanza un nivel medio de pensamiento crítico en la técnica de manejo de cateterismo vesical. En cuanto a las ocho dimensiones que abarcó el estudio, se evidencia que en agudeza perceptiva y cuestionamiento permanente, alcanzan un nivel medio de pensamiento crítico con $55,6 \%$ y $57,8 \%$, respectivamente; en tanto, en coraje intelectual, mente abierta, autorregulación, control emotivo y valoración justa, lograron un nivel alto de pensamiento crítico con $53,3 \%, 84,4 \%, 71,1 \%, 53,3 \%$ y $80,0 \%$, respectivamente; finalmente, en construcción y reconstrucción de los saberes, el $66,7 \%$ obtuvo un nivel bajo de pensamiento crítico en la técnica de manejo de cateterismo vesical. Conclusiones: El nivel de pensamiento crítico de los profesionales de enfermería en la técnica de manejo de cateterismo vesical, es de nivel medio.
\end{abstract}

Palabras clave: Conocimiento, pensamiento crítico, cateterismo, sondaje vesical.

\section{SUMMARY}

Objective: Determine the level of critical thinking of nursing professionals in the art of managing bladder catheterization in the emergency services, hospitalization, medical and surgical Vitarte Hospital. Material and Methods: The research design was quantitative, descriptive, cross-sectional. The sample consisted of 45 nurses (I) emergency services (25), hospital medicine (12) and surgery (8) Vitarte Hospital. Using the instrument, "Critical Thinking Questionnaire," which consisted of 39 specific questions, the same that was validated by experts. Reliability test was 0,803 according to the Cronbach alpha coefficient. Results: The study showed that $100 \%$ of the sample, $64.4 \%$ of nurses, reaches an average level of critical thinking in management technique catheterization. As for the eight dimensions covered by the study, it appears that in perceptual acuity and constant questioning, reach an average level of critical thinking with $55.6 \%$ and $57.8 \%$, respectively, while in intellectual courage, open mind, self-regulation, emotional control and fair assessment, achieved a high level of critical thinking with $53.3 \%, 84.4 \%, 71.1 \%, 53.3 \%$ and $80.0 \%$, respectively, finally, in construction and reconstruction of knowledge, $66.7 \%$ had a low level of critical thinking in management technique catheterization. Conclusions: The level of critical thinking of nursing professionals in the art of managing bladder catheterization, is average.

Keywords: Knowledge, critical thinking, catheterization, urinary catheterization

${ }^{1}$ Enfermera asistencial del servicio de pediatría clínica Ricardo Palma, San Isidro, Perú.

${ }^{2}$ Profesor auxiliar de la EAP de Enfermería, Facultad de Ciencias de la Salud, Universidad Peruana Unión, Lima, Perú. 


\section{INTRODUCCIÓN}

El informe de la Organización de las Naciones Unidas para la Educación, la Ciencia y la Cultura (UNESCO) generado por el equipo de Delors (1996), plantea potenciar la comprensión, despertar la curiosidad intelectual, estimular el sentido crítico y adquirir al mismo tiempo autonomía de juicio.

Paul y Elder (2005), afirman que el pensamiento crítico se está volviendo cada vez más importante debido a cuatro tendencias: cambio acelerado, aumento en complejidades, intensificación de la interdependencia e incremento del peligro.

Paul y Elder (2003), refieren que todo el mundo piensa, es parte de nuestra naturaleza. Pero, mucho de nuestro pensar, por sí solo, es arbitrario, distorsionado, parcializado, desinformado o prejuiciado. Sin embargo, nuestra calidad de vida y de lo que producimos, hacemos o construimos depende, precisamente, de la calidad de nuestro pensamiento. El pensamiento de mala calidad cuesta tanto en dinero como en calidad de vida. La excelencia en el pensamiento, sin embargo, debe ejercitarse de forma sistemática.

Lo mencionado con anterioridad, evidencia que el pensamiento crítico es propio del ser humano. Por otra parte, la sociedad de hoy exige al individuo adoptar una postura crítica frente a los eventos que cotidianamente debe afrontar, en tal sentido, dada las características de la sociedad actual, es cada vez mayor la necesidad de intervenir rápida y eficazmente en la adquisición de habilidades de pensamiento y procesos (Pitchers \& Soden, 2000).

Cada vez, el mundo se hace más cambiante y complejo. Ante este panorama se hace necesario que las personas se adapten, por lo que no basta tener mayores conocimientos que también están sujetos a dicho cambio, sino además la habilidad para aplicarlos con éxito, es decir, la habilidad de pensar con efectividad (Betancourt, 2004).

Del mismo modo, Uzeta (2009) refiere que la naturaleza del pensamiento crítico es una actitud y un proceso de razonamiento que envuelve un número de destrezas intelectuales, una actividad mental con propósito en el cual las ideas son producidas y evaluadas. Se caracteriza por su conceptualización al hacer una imagen mental de lo que se está pensando, de manera racional y razonable, basado en la razón más que en prejuicio, preferencia, interés propio o miedos; es reflexivo, significa que la persona que piensa críticamente no pasa a conclusiones o hace decisiones apresuradas.

Por tanto, la naturaleza de necesidades de cuidado de enfermería, permite que el personal de enfermería tenga en sus manos, una proporción significativa de procesos relacionados con el pensamiento crítico, como profesional, en la colocación de sonda vesical, donde también interviene el paciente; muchos aspectos están ligados al comportamiento humano del personal, paciente y equipos de salud. El profesional de enfermería necesita contar con una base que indique los presupuestos de comportamiento del que dispone tales como activos, reflexivos, teórico o pragmáticos o las variantes en las combinaciones de ellos, para generar preferencias de estilos de intervención. Pues, el futuro pertenece a aquellos que aprendan a obtener el máximo de su poder mental, a aquellos que piensen críticamente. Para tener éxito en el mundo actual rápidamente cambiante se debe tener más conocimientos que los usuales relacionados con el trabajo. Se debe precisar de habilidades de pensamiento altamente desarrolladas, habilidades de pensamiento crítico, que ayuden a adaptarse a las nuevas situaciones, tomar decisiones competentes y aprender a aprender. En tal sentido, Uzeta (2009), refiere que Enfermería es una disciplina, un campo de estudio que demanda ejecutar, no solo adquirir conocimiento. Esas acciones que ejecuta y las decisiones que toma pueden afectar profundamente la vida de otros, ya que los cambios rápidos en los escenarios de trabajo de enfermería son importantes y obligan a estos profesionales pensar críticamente.

En cuanto al uso de sondas vesicales, Warren, citado por Smithson (2008), afirma que las infecciones urinarias en pacientes portadores de sonda urinaria son las infecciones nosocomiales más frecuentes, tanto en hospitales como en centros de larga estancia. En relación a lo mencionado, Rubin, Tolkoff-Rubin y Cotran (1991), expresan que la infección del tracto urinario es la forma más común de infección bacteriana humana. De la misma manera, Foxman (2003), afirma que este tipo de infección es una de las infecciones bacterianas más frecuentemente diagnosticadas.

Hernández (2010), manifiesta que la infección del tracto urinario (ITU) se caracteriza por la presencia de microorganismos en el tracto urinario a cualquier nivel, desde el extremo distal de la uretra hasta el corte renal (uretra, vejiga, próstata, uréteres, pelvis renal o riñones), englobando diferentes entidades clínicas que requieren su catalogación mediante la correlación clínica-laboratorio.

Respecto a lo mencionado con anterioridad, Martínez y col. (2000), afirman que la infección del tracto urinario ocupa en promedio el tercer lugar entre las infecciones nosocomiales en Norteamérica, España y varios países de América Latina; representa entre el 23 al $30 \%$ del total de infecciones adquiridas 
en el hospital, aumenta la estancia 4 días como media e incrementa los costos de la atención en grado considerable.

Asimismo, las infecciones urinarias nosocomiales según el Ministerio de Salud de Chile (MINSAL, 2007) son una de las complicaciones más frecuentes de la hospitalización, siendo responsable de más del $40 \%$ del total de infecciones adquiridas en los hospitales. El 10\% de los pacientes hospitalizados son sometidos a una cateterización vesical y de ellos alrededor del 10\% padecerán una infección urinaria. Cerca del $80 \%$ se genera por instrumentación del tracto urinario principalmente por cateterismo urinario permanente.

De acuerdo al Ministerio de Salud del Perú (MINSA, 2010) en el año 2008 las enfermedades del aparato urinario, en general, ocupa el octavo lugar con un porcentaje de $3,8 \%$; en los jóvenes ocupa el sexto lugar con $6,4 \%$; en los adultos, el quinto lugar con 7,3\%; en el adulto mayor, se ubica en el séptimo puesto con $5,6 \%$, como causa de morbilidad según las atenciones de consulta externa realizadas en los establecimientos de salud del MINSA. En el año 2007, las infecciones urinarias se ubicaron dentro de las principales causas de mortalidad por causas específicas con una tasa de 32,8 ; no se tiene información estadística precisa respecto a ITU causado por sonda vesical.

En tanto, en el Hospital Vitarte, las infecciones urinarias como causas de morbilidad en hospitalización medicina en el año 2011, ocupó el sexto lugar con un $3.26 \%$; en el servicio de hospitalización pediátrica, se ubica el quinto lugar con $4.30 \%$; asimismo, en el servicio de emergencia de medicina se presentaron 184 casos y en el servicio de emergencia pediátrica 73 casos durante el año. En cuanto a la estancia hospitalaria, se tiene en promedio en los servicios de medicina interna 4,9 días, en cirugía 3,9 días y en pediatría 3,7 días.

En el mencionado hospital, no se encuentran datos específicos en cuanto a ITU causado por cateterismo vesical, no obstante, al realizar una entrevista verbal a profesionales y no profesionales de salud de dicho nosocomio, las respuestas coinciden. "El sondaje vesical es uno de los procedimientos que con frecuencia se realiza, al igual que la colocación de sonda nasogástrica. Dichos procedimientos al igual que otros, se intentan realizar lo más aséptico posible, pero esto se ve vulnerado por algunos factores como: poco abastecimiento de insumos y materiales, no se cuenta con ambiente especial para procedimientos, los ambientes de trabajo son reducidos, hay demasiada demanda y el hacinamiento es alarmante; el Seguro
Integral de Salud (SIS) reconoce una sola vez los materiales, recursos económicos bajos de los pacientes para la adquisición de sondas u otros materiales para recambios, ejecución de procedimientos mecanizados (robotizados) y otros".

Barrasa y Aspiroz (1996), sostienen que cerca del $30 \%$ de las infecciones del tracto urinario se puede disminuir con la aplicación de medidas higiénicas y sanitarias, suficientemente estandarizadas, porque han demostrado ser eficaces en el control de los factores de riesgo intrínsecos relacionados con el paciente y extrínsecos relacionados con el cateterismo y la antibioticoterapia que altera a flora existente.

En tal sentido, afirmamos que las enfermeras (os) deben tomar decisiones complejas, adaptarse a nuevas situaciones y actualizar continuamente sus conocimientos y habilidades. El pensamiento crítico forma parte integral de todo ello. El pensamiento crítico es la clave para prevenir y resolver los problemas. Los profesionales de enfermería que no piensen de forma crítica se convierten en parte del problema (Alfaro-LeFevre, 2009).

La investigación aporta a los profesionales y estudiantes de enfermería información necesaria acerca del conocimiento y dimensiones necesarias que el cuidador deberá tener en el momento de realizar la colocación de sonda vesical, haciendo uso del pensamiento crítico para su ejecución, a fin de mejorar esta técnica.

Para lograr este ideal es necesario un cuidado de calidad, continuo, oportuno y humano, de esta manera se logrará contribuir a la disminución de negligencias en la realización de la técnica de colocación de sonda vesical. Murillas (2010), menciona que el futuro pertenece a aquellos que aprendan a obtener el máximo de su poder mental, a aquellos que piensen críticamente. Para tener éxito en el mundo actual rápidamente cambiante se debe tener más conocimientos que los usuales relacionados con el trabajo. Se precisa de habilidades de pensamiento altamente desarrollado, habilidades de pensamiento crítico, que ayuden a adaptar a los profesionales a las nuevas situaciones, para tomar decisiones competentes que mejorarán la atención brindada por el profesional de enfermería.

Garza y De la Garza (2010), afirman: pensar críticamente facilita el estudio de las diferentes disciplinas de conocimiento para procesar datos, observar con cuidado, analizar, sintetizar, plantear conclusiones, relacionar ideas, formular hipótesis, diseñar modelos explicativos, etcétera. Además, pensar de forma crítica hace a las personas más 
Nivel de pensamiento crítico de los profesionales de enfermería en la técnica de manejo de cateterismo vesical en los servicios de emergencia, hospitalización de medicina y cirugía del Hospital Vitarte

competitivas y ofrece mayores posibilidades de éxito en un futuro, en el ámbito laboral y de la vida cotidiana en general.

Considerando que el propósito de enfermería es garantizar seguridad, eficacia y continuidad en sus cuidados, así como ciertas condiciones que incrementan los riesgos, como colocación del cateterismo vesical y las consecuencias que este puede generar, motivó el interés por conocer el nivel de pensamiento crítico de los profesionales de enfermería en la técnica de manejo de colocación de sonda vesical.

El objetivo principal del estudio fue determinar el nivel de pensamiento crítico de los profesionales de enfermería en la técnica de manejo de cateterismo vesical en los servicios de emergencia, hospitalización de medicina y cirugía del Hospital Vitarte.

\section{MATERIAL Y MÉTODOS}

El estudio se realizó en el Hospital Vitarte, en Lima (Perú), durante el año 2013. El diseño de investigación es cuantitativo. Es descriptivo, porque se indaga los niveles de pensamiento crítico de los profesionales de enfermería en la técnica de colocación de sonda vesical; de corte transversal, porque este tipo de investigación tiene como característica recolectar datos e información en un único momento. La muestra estuvo conformada por 45 enfermeras(os); de los servicios de emergencia (25), hospitalización de medicina (12) y cirugía (8), del Hospital Vitarte. Se utilizó el instrumento "Cuestionario de Pensamiento Crítico" elaborado por la autora, el cual constó de 39 preguntas específicas, el mismo que fue validado por expertos y la prueba de confiabilidad fue de 0,803 según el Coeficiente Alfa de Cronbach, siendo ejecutado por la investigadora en el mes de junio de 2013.

\section{Criterios de inclusión:}

- Enfermeras(os) nombradas(os) y/o con Contrato Administrativo de Servicios (CAS) que laboren en los servicios donde se realiza el estudio.

- Enfermeras(os) con voluntad de participar en el estudio.

\section{Criterios de exclusión:}

- Enfermeras(os) que no acepten participar en el estudio.

- Enfermeras(os) que estén de vacaciones.

- Enfermeras(os) que estén realizando pasantías.

La recolección de datos se realizó a través de una encuesta elaborada por la investigadora, previamente validada por expertos. La encuesta estuvo constituida por dos secciones, las cuales fueron: Datos generales (Edad, género, especialidad, tiempo de servicio, área en la que labora). Información específica, constituida por ocho dimensiones: Agudeza perceptiva, cuestionamiento permanente, construcción y reconstrucción de los saberes, coraje intelectual, mente abierta, autorregulación, control emotivo y valoración justa.

El instrumento fue aplicado previa autorización de la institución y de las enfermeras (os) con voluntad de participación, luego de explicarse el motivo del cuestionario en forma verbal; la encuesta fue ejecutada por la investigadora. La información fue procesada con el paquete estadístico SPSS versión 19.0.

\section{RESULTADOS}

Tabla 1

Nivel de pensamiento crítico de los profesionales de enfermería según dimensiones en la técnica de manejo de cateterismo vesical en los servicios de emergencia, hospitalización de medicina y cirugía del Hospital Vitarte, junio - 2013.

\begin{tabular}{|c|c|c|c|c|c|c|}
\hline \multirow{3}{*}{ Dimensiones } & \multicolumn{6}{|c|}{ Nivel de pensamiento crítico } \\
\hline & \multicolumn{2}{|c|}{ Bajo } & \multicolumn{2}{|c|}{ Medio } & \multicolumn{2}{|c|}{ Alto } \\
\hline & $\mathrm{n}$ & $\%$ & $\mathrm{n}$ & $\%$ & $\mathrm{n}$ & $\%$ \\
\hline Agudeza perceptiva & 10 & 22,2 & 25 & 55,6 & 10 & 22,2 \\
\hline Cuestionamiento permanente & 16 & 35,6 & 26 & 57,8 & 3 & 6,7 \\
\hline Construcción y reconstrucción de los saberes & 30 & 66,7 & 12 & 26,7 & 3 & 6,7 \\
\hline Coraje intelectual & 0 & 0 & 21 & 46,7 & 24 & 53,3 \\
\hline Mente abierta & 0 & 0 & 7 & 15,6 & 38 & 84,4 \\
\hline Autorregulación & 0 & 0 & 13 & 28,9 & 32 & 71,1 \\
\hline Control emotivo & 0 & 0 & 21 & 46,7 & 24 & 53,3 \\
\hline Valoración justa & 0 & 0 & 9 & 20,0 & 36 & 80,0 \\
\hline
\end{tabular}


De acuerdo a la tabla 1 , se puede evidenciar que del $100 \%$ (45) de enfermeras, el $55,6 \%$ posee un nivel medio de pensamiento crítico en la dimensión de agudeza perceptiva, mientras que el $22,2 \%$ alcanza un nivel bajo y con igual porcentaje $(22,2 \%)$ logra un nivel alto; en la dimensión de cuestionamiento permanente el 57,8\% logra alcanzar un nivel medio, seguido de un nivel bajo con $35,6 \%$ y solo el $6,7 \%$ posee un nivel alto; en cuanto a construcción y reconstrucción de los saberes, el $66,7 \%$ de las enfermeras posee un nivel bajo y solo el $6,7 \%$ alcanza un nivel alto; asimismo, en la dimensión de coraje intelectual el 53,3\% de la muestra obtiene un nivel alto y el $46,7 \%$ un nivel medio; de la misma manera, en la dimensión de mente abierta el $84,4 \%$ posee nivel de pensamiento crítico alto; por otro lado, en la dimensión autorregulación el $71,1 \%$ tiene un nivel de pensamiento crítico alto y el $28,9 \%$ un nivel medio; en tanto, respecto a control emotivo el $53,3 \%$ posee un nivel de pensamiento crítico alto y $46,7 \%$ nivel medio; finalmente, el 80,0\% logra tener un nivel alto de pensamiento crítico en la valoración justa y el 20,0\% un nivel medio; todo ello en la técnica de manejo de cateterismo vesical en los servicios de emergencia (medicina, cirugía, pediatría y trauma shock) y hospitalización de medicina y cirugía del Hospital Vitarte.

Tabla 2

Nivel de pensamiento crítico de los profesionales de enfermería en la técnica de manejo de cateterismo vesical en los servicios de emergencia, hospitalización de medicina y cirugía del Hospital Vitarte, junio - 2013.

\begin{tabular}{lll}
\hline Nivel & $\mathrm{n}$ & $\%$ \\
\hline Alto & 16 & 35,6 \\
Medio & 29 & 64,4 \\
Bajo & 0 & 0 \\
Total & 45 & 100 \\
\hline
\end{tabular}

La tabla 2 muestra que el 64,4\% de las enfermeras encuestadas presenta un nivel medio de pensamiento crítico y el 35,6\%, un nivel alto de pensamiento crítico en la técnica de manejo de cateterismo vesical.

\section{DISCUSIÓN}

El presente trabajo de investigación muestra que el $55,6 \%$ de las enfermeras(os) en estudio tiene un nivel medio de pensamiento crítico en la dimensión de agudeza perceptiva, esto es, que los profesionales en cierta medida dedican un tiempo para poder observar detalles en cuanto a la cateterización vesical, por lo que el profesional de enfermería necesita asegurarse de la precisión de la información y de su accionar. Keating, (2006), menciona que el profesional de enfermería debe priorizar, gestionar y justificar sus acciones a través de la agudeza perceptiva, observando mínimos detalles en cada procedimiento.

En cuanto a la dimensión Cuestionamiento Permanente, se obtiene que el 35,6\% tiene nivel bajo y $57,8 \%$, nivel medio. Estos datos reflejan que los profesionales de enfermería no están cuestionando aspectos importantes que tienen que ver con el procedimiento de cateterismo vesical, las acciones realizadas están siendo monótonas, robóticas $\mathrm{o}$ mecanizadas, lo cual puede ser perjudicial para el paciente; al respecto, Paul y Elder (2003), afirman que la atención brindada de manera mecanizada o monótona, afecta la recuperación pronta del paciente y esto cuesta tanto en dinero como en calidad de vida. Del mismo modo, Uzeta (2009), refiere que esas acciones que ejecuta y las decisiones que toma el profesional de enfermería pueden afectar profundamente la vida de otros por lo que necesitan pensar críticamente.

En la dimensión de construcción y reconstrucción de los saberes muestra que el $66,7 \%$ de los profesionales de enfermería tiene un nivel bajo, esto quiere decir que los profesionales de enfermería no están incorporando nuevos conocimientos que ayuden a brindar cuidados de manera óptima (Aguilera, Zubizarreta \& Castillo, 2006). Es decir, se necesita procesar y modificar sus conocimientos de ser necesario, de tal manera que relacione dialécticamente la teoría y la práctica, con el fin supremo de prestar atención de calidad al paciente.

Por otro lado, el 53,3\% presenta un nivel alto de pensamiento crítico en relación a la dimensión de Coraje Intelectual, es decir que este grupo afronta con entereza y decisión situaciones difíciles que se logran dar cuando se realiza el procedimiento de cateterismo vesical; asimismo, en otros contextos del ejercicio de la profesión, ante otras disciplinas, temas o trabajos en equipo expone con altura sus planteamientos. En cualquier medio que labora, sea 
pasivo o activo, apacible o conflictivo la enfermera se mantiene firme ante las críticas y no se doblega ante la injuria ni cae en la tentación de reaccionar en forma negativa. Cuando observan acciones que son incorrectas, falta a la ética profesional, ellas dicen las cosas con objetividad y altura, sin amedrentarse por los prejuicios. Paul y Elder (2005), mencionan que el coraje intelectual de un profesional de enfermería está basado en el conocimiento de la necesidad de enfrentar y de abordar con justicia las ideas, creencias o puntos de vista hacia los cuales uno tiene fuertes emociones negativas y hacia los que no han sido seriamente considerados. Del mismo modo, implica la voluntad para enfrentar la desaprobación del grupo al expresar una idea que no sea popular o desafiar una que sí lo sea.

En la dimensión de Mente Abierta el profesional de enfermería obtuvo un nivel alto $(84,4 \%)$, es decir, escuchan nuevas ideas y puntos de vista, y consideran la situación desde diversas perspectivas, tienen tolerancia con los puntos de vista divergentes. El pensamiento crítico del enfermero es deliberado, dirigido a un objetivo, emite juicios basados en evidencias, se basa en principios de la ciencia y del método científico; por otra parte, requiere estrategias que maximicen el potencial humano y compense los problemas causados por la naturaleza humana. Estos aspectos necesitan de una mente abierta, que sea receptiva al conocimiento, se adecúe a los cambios en el avance de la ciencia y tecnología, y entienda la naturaleza humana en sí en sus dimensiones física, mental, social y espiritual; además, hay situaciones comunes en las que el enfermero utiliza el pensamiento crítico, así como la comprensión de algo o de alguien, identificación de problemas reales o potenciales, determinación de resultados deseables, toma de decisiones en un plan de acción, hallar formas de mejora, etcétera, lo cual también implica el uso de la capacidad de mente abierta (Facione, 2007). Frente a lo mencionado, Añorve (2008), afirma que el profesional de enfermería piensa con mente abierta dentro de sistemas alternos de pensamiento, reconociendo y evaluando, según sea necesario, los supuestos, implicaciones y consecuencias prácticas de estos; asimismo, Vásquez (2009), menciona que los profesionales que están con el paciente tienen la responsabilidad de ir avanzando y creciendo en el desarrollo de la disciplina de enfermería, manteniendo una actitud positiva y una mente abierta a la innovación y seguir trabajando para ser profesionales competentes.

El 71,1\% de los profesionales de enfermería que lograron un nivel de pensamiento crítico alto, en la dimensión de autorregulación, tienen la capacidad de análisis, inferencia, interpretación, explicación y evaluación que, sustentada por la autorregulación, se convierte en lo que distingue al profesional de enfermería que está capacitado para enfrentar los desafíos de la sociedad moderna, pues el mundo actual, que es de gran complejidad, demanda de un pensamiento de alta calidad. Por ende, los pensadores críticos encarnan el principio socrático: Una vida sin examinarse no vale la pena vivir, porque se dan cuenta de que muchas vidas no examinadas en conjunto dan como resultado un mundo peligroso, sin sentido crítico e injusto (Paul \& Elder, 2008). Por otro lado, el $28,9 \%$ tiene un nivel medio de autorregulación. La excesiva carga mental, la mala organización, la presión asistencial, la complejidad de las técnicas o los turnos de trabajo llevan a un desgaste personal incuestionable. No es menos cierto, que el desgaste que genera en el enfermero, el fingir emociones positivas o desviar y refrenar las negativas constantemente, y la autorregulación emocional a la que se someten para no caer en el abismo; es así que las enfermeras, en cierta medida, necesitan reflexionar sobre sus acciones y tomar en positivo lo negativo, además tener conciencia de sus fortalezas y limitaciones (Paul \& Elder, 2003).

Añorve (2008), menciona que el pensamiento crítico implica comunicarse efectivamente, desarrollar habilidades para la solución de problemas y el compromiso de superar el egocentrismo del ser humano. Las estadísticas de la presente investigación evidencian que más del $50 \%(53,3 \%)$ de las enfermeras sabe mantener la calma ante las ideas o pensamientos contrarios a las de ellas, son críticos ante las propuestas pero nunca ante los que lo plantean, reúnen y evalúan la información, se comunican efectivamente con otros para idear soluciones a problemas complejos e interactuar con los demás de forma efectiva (Ministerio de Educación, 2004). Según Kurland, D.J., citado por Uzeta (2009), en sentido amplio, pensar críticamente está relacionado con la razón, la honestidad intelectual y la amplitud mental en contraposición a lo emocional, a la pereza intelectual y a la estrechez mental.

Los profesionales de enfermería en estudio objetivamente dan valor justo a las opiniones o acometimientos en un $80 \%$, esto implica que cada profesional cumple su rol con actitud justa. León (2006), afirma que la valoración justa es el talento para otorgar a sus opiniones y suceso el valor que objetivamente se merece, sin dejarse influenciar por los sentimientos y emociones.

El nivel de pensamiento crítico de los profesionales de enfermería en la técnica de manejo de cateterismo vesical en los servicios de emergencia (medicina, 
cirugía, pediatría y trauma shock), hospitalización de medicina y cirugía del Hospital Vitarte, alcanza un nivel medio con un $64,4 \%$; en cuanto a este resultado, Aguilera (2006), afirma que para muchos enfermeros y enfermeras, el pensamiento crítico significa simplemente buen juicio clínico o solución efectiva del problema; para tener éxito en el campo del cuidado de la salud se necesita de una visión más amplia: no se puede quedar satisfecho solo con tener una mentalidad de solución de problemas. Podrá demostrar buen juicio clínico y solución de problemas, pero si se tiene un deseo sincero de mejorar, de encontrar la forma de ampliar sus conocimientos y habilidades, y de hacer la práctica eficiente y eficaz, se está pensando críticamente. En cuanto a este resultado obtenido, Alfaro-LeFevre (1997), menciona la importancia que tiene el desarrollo del pensamiento reflexivo, cuando consideramos que las enfermeras, dada la naturaleza de sus actividades profesionales, deben tomar decisiones complejas, adaptarse constantemente a nuevas situaciones y actualizar continuamente sus conocimientos y habilidades, y que el pensamiento crítico forme parte integral de todo ello.

En cuanto a la dimensión de agudeza perceptiva y cuestionamiento permanente, los profesionales de enfermería alcanzan un nivel medio de pensamiento crítico.

Asimismo, en la dimensión de construcción y reconstrucción de los saberes, los profesionales de enfermería alcanzan un nivel bajo de pensamiento crítico y en la dimensión de coraje intelectual, mente abierta, autorregulación, control emotivo y valoración justa, alcanzan un nivel alto de pensamiento crítico cuando se realiza el procedimiento de cateterismo vesical.

Por otro lado, el pensamiento crítico en enfermería permite focalizar la valoración, emitir diagnósticos enfermeros y sustentar las demás etapas del Proceso de Atención de Enfermería basado en la evidencia, y corresponde a ellas tomar conciencia de la importancia que este tiene y los beneficios que aportará para el desarrollo disciplinario y profesional de la enfermería.

Finalmente, se concluye que el nivel de pensamiento crítico de los profesionales de enfermería en la técnica de manejo de cateterismo vesical, es de nivel medio.

\section{Declaración de financiamiento y de conflicto de intereses:}

El estudio fue financiado por la autora, quien declara no tener algún tipo de conflicto de interés en la investigación realizada.

\section{Correspondencia:}

Luz Yanet Barboza Núñez

Correo electrónico: lybarnu_308@hotmail.com.

\section{REFERENCIAS BIBLIOGRÁFICAS}

Álvarez, M. \& Fernández, R. (1999). Propuesta de un programa de métodos de estudio. Madrid. Tea Ediciones.

Ballenato, P. (2005). Técnicas de Estudio: el aprendizaje activo y positivo. Madrid: Editorial Pirámide.

Barrena, S. (2001). Los hábitos y el crecimiento: una perspectiva peirceana. Revista electrónica razón $y$ palabra, 21(4), 2-4. Recuperado de: www. razonypalabra.org.mx/anteriores/n21/21_sbarrena.html

Bazán, J. \& Aparicio, A. (2001). Modelo explicativo de las relaciones entre hábitos de estudio, sexo y procedencia en egresados de secundaria de nivel socioeconómico bajo, (Perú). Revista de Investigación en Psicología, 4(2), 41-53. Recuperado de: http://sisbib.unmsm.edu. pe/bvrevistas/investigacion_psicologia/v04_n2/pdf/ a04v4n2.pdf.pdf

Benito, D.; Lorenzo, N. (2002). Técnicas de estudio. Barcelona: Editorial Océano.
Belaúnde, I. (1994). Hábitos de Estudio. En: Revista de la Facultad de Psicología de la Universidad Femenina del Sagrado Corazón, año 2, № 2, octubre, Lima.

CONEAU. (2012). Presentan diagnóstico de la calidad educativa en el Perú. Revista electrónica Actualidad. Recuperado de: http://www.generaccion.com/ noticia/147186/presentan-diagnco-situacie-calidadeducaciuperior-per

Cartagena, M. (2008). Relación de la autoeficacia y el rendimiento escolar y los hábitos de estudio en alumnos de secundaria, (Perú). Revista Iberoamericana sobre Calidad, Eficacia y Cambio en Educación. 6(3), 60-99. Recuperado de: http://www.rinace.net/arts/vol6num3/ art3.pdf

Calero, M. (1992). Técnicas de estudio e investigación. Lima: Editorial San Marcos.

Castillo; A.; Polanco; L. (2005). Enseña a estudiar. Aprende a aprender. Didáctica del estudio. Madrid: Pearson Educación S.A. 
Nivel de pensamiento crítico de los profesionales de enfermería en la técnica de manejo de cateterismo vesical en los servicios de emergencia, hospitalización de medicina y cirugía del Hospital Vitarte

Congrains, E. (1991). Así es como se estudia. Bogotá: Editorial Forja de Colombia.

Díaz, M. (1998). Método de Estudio. Lima: Edit. De libros técnicos Elite.

Díaz, J. (1990). Aprende a estudiar con éxito. México: Editorial Trillas.

Díaz, M.; Peio, A.; Escudero, T.; Rodríguez, S.; Vidal, G. (2002). Evaluación del rendimiento académico en la enseñanza superior. Comparación de resultados entre alumnos procedentes de la LOGSE y del COU. Revista de investigación educativa, 2(20), 357,383.
Escalante, L., Escalante Y., Linzaga C. \& Merlos M. (2008). Comportamiento de los estudiantes en función a sus hábitos de estudio. Actualidades Investigativas en Educación. 8(2), 1-15.

Recibido: $15 / 06 / 2013$

Aceptado: 20/11/2013 